Theorems 1 and 2 have their analogues in the case of integrated Lipschitz condition; that is, the following theorem holds:

Theorem 3. If $f(x) \in \operatorname{Lip}(\alpha, p), 0<\alpha<1, p \geqq 1$, then for any $\beta>\alpha$

$$
\left(\int_{0}^{1}\left|\sigma_{n}^{(\beta)}(x ; f)-f(x)\right|^{p} d x\right)^{1 / p}=O\left(n^{-\alpha}\right) .
$$

Since the proof is analogous to the preceding one, we shall omit it.

TôHoKU UNIVERSITY

\title{
POSITIVE INFINITIES OF POTENTIALS
}

\section{WALTER RUDIN}

Let $R$ denote Euclidean 3-space. The following theorem is due to Evans [1, p. 421]. ${ }^{1}$

Let $E$ be a closed bounded set of capacity zero in $R$. There exists a distribution of positive mass $\mu(e)$ entirely on $E$, such that its potential $V(M)=\int_{R}(1 / M P) d \mu(P)$ is infinite at every point of $E$ and at no other points.

A proof of the two-dimensional analogue was published by Noshiro [2]. In the present note we show, by a modification of Evans' construction, that an absolutely continuous distribution exists whose potential is infinite on the preassigned set $E$ only. More precisely, our result, extended to unbounded sets, is as follows:

Theorem. Let $E$ be a closed set of capacity zero in $R$, and let $G$ be an open set containing $E$. Then there exists a non-negative function $f$ which is summable on $R$, such that the superharmonic function (that is, the potential)

$$
F(M)=\int_{R} \frac{1}{M P} f(P) d P
$$

is infinite on $E$, is continuous in $R-E$, and is harmonic in $R-\bar{G}$. ( $\bar{G}$ denotes the closure of $G$.)

Analogous results evidently hold in two and in more than three dimensions.

Presented to the Society, February 24, 1951; received by the editors December 4, 1950.

${ }^{1}$ Numbers in brackets refer to the references at the end of the paper. 
To begin with, we suppose that $E$ is bounded and contains infinitely many points. Let $n$ be a positive integer, and put

$$
V_{n}=\max _{Q_{1}, \cdots, Q_{n} \in E}\left\{\min _{P \in E} \frac{1}{n}\left(\frac{1}{P Q_{1}}+\cdots+\frac{1}{P Q_{n}}\right)\right\} .
$$

Since $E$ contains infinitely many points, $V_{n}<+\infty$. The compactness of $E$ implies that there exist points $P_{1}, \cdots, P_{n}$ on $E$, such that, for all $P$ on $E$,

$$
\frac{1}{n}\left(\frac{1}{P P_{1}}+\cdots+\frac{1}{P P_{n}}\right) \geqq V_{n} .
$$

The transfinite diameter of $E$ is defined as the limit of the sequence $\left\{D_{n}\right\}$ where

$$
\frac{n(n-1)}{2} \cdot \frac{1}{D_{n}}=\min _{Q_{1}, \cdots, Q_{n} \in E}\left\{\sum_{1 \leqq i<k \leqq n} \frac{1}{Q_{i} Q_{k}}\right\} .
$$

It can be shown $[1$, p. 423$]$ that $V_{n} \geqq\left(D_{n+1}\right)^{-1}$. The transfinite diameter of a compact set being equal to its capacity [3], it follows that

$$
\lim _{n \rightarrow \infty} V_{n}=+\infty \text {. }
$$

So far we have followed Evans. We now choose $r_{n}$ such that $0<r_{n}<\left(n V_{n}\right)^{-1}$ (this is possible, since $V_{n}<+\infty$ ), and such that the closed spheres $S_{i}$ with centers at $P_{i}(i=1, \cdots, n)$ and radius $r_{n}$ are contained in $G$. For $i=1, \cdots, n$, we define

$$
\begin{aligned}
& \phi_{i}(P)=\left\{\begin{array}{rr}
\frac{3}{4 n \pi r_{n}^{3}} & \left(P \in S_{i}\right), \\
0 & \left(P \in R-S_{i}\right),
\end{array} \quad u_{n}(P)=\sum_{i=1}^{n} \phi_{i}(P) \quad(P \in R),\right. \\
& U_{n}(M)=\int_{R .} \frac{1}{M P} u_{n}(P) d P \quad(M \in R) .
\end{aligned}
$$

Then $\int_{R} u_{n}(P) d P=1$, and $U_{n}$ is the potential of a unit mass. Suppose $M \in E$. If $M \in R-\left(S_{1}+\cdots+S_{n}\right)$, then

$$
U_{n}(M)=\frac{1}{n}\left(\frac{1}{M P_{1}}+\cdots+\frac{1}{M P_{n}}\right) \geqq V_{n}
$$

(by (2)). If $M \in S_{j}$, then

$$
U_{n}(M)>\int_{S_{j}} \frac{1}{M P} \phi_{j}(P) d P=\frac{3 r_{n}^{2}-t^{2}}{2 n r_{n}^{3}} \geqq \frac{1}{n r_{n}}>V_{n},
$$


where $t=M P_{j}$. By (4), (5),

$$
U_{n}(M) \geqq V_{n}
$$

By (3), there is a sequence $\left\{n_{k}\right\}$ such that $V_{n_{k}} \geqq 2^{k}$. We define

$$
\begin{aligned}
f(P) & =\sum_{k=1}^{\infty} 2^{-k} \boldsymbol{U}_{n_{k}}(P) & & (P \in R), \\
F(M) & =\int_{R} \frac{1}{M P} f(P) d P=\sum_{k=1}^{\infty} 2^{-k} U_{n_{k}}(M) & & (M \in R) .
\end{aligned}
$$

Then $\int_{R} f(P) d P=1$; by (6), $F(M)=+\infty$ if $M \in E$; if $M \in R-E, f$ is bounded in some neighborhood of $M$ (since $r_{n} \rightarrow 0$ as $n \rightarrow \infty$ ), which implies that $F$ is continuous in $R-E$; and in $R-\bar{G}, f=0$, hence $F$ is harmonic.

Next, if $E$ is finite, let $E=A_{1}+\cdots+A_{m}$. Choose $r>0$ such that the closed spheres $S_{i}$ with centers at $A_{i}(i=1, \cdots, m)$ and radius $r$ are contained in $G$, and define

$$
\phi(t)=\left\{\begin{array}{lr}
t^{-2} & \text { if } 0<t<r, \\
0 & \text { otherwise, }
\end{array} \quad f(P)=\sum_{i=1}^{m}\left(\phi P A_{i}\right) \quad(P \in R) .\right.
$$

The conclusion of the theorem evidently holds for this function $f$. Hence the theorem is proved for bounded sets $E$.

Finally, suppose $E$ is unbounded. There exist compact sets $E_{i}(i=1,2,3, \cdots)$ such that $R=\sum_{i=1}^{\infty} E_{i}$, and open sets $G_{i}$ containing $E_{i}$ such that no point of $R$ is in more than four of the sets $G_{i}$. We now apply the previously obtained result for bounded sets to construct functions $f_{i}(i=1,2,3, \cdots)$ which satisfy the conclusion of the theorem with respect to the sets $E \cdot E_{i}$ and $G \cdot G_{i}$, such that $\int_{R} f_{i}(P) d P=2^{-i}$, and put $f(P)=\sum_{i=1}^{\infty} f_{i}(P)$. For any $P$, this sum contains at most four nonzero terms. Hence it is easily verified that the conclusion of the theorem holds.

\section{REFERENCES}

1. G. C. Evans, Potentials and positively infinite singularities of harmonic functions, Monatshefte für Mathematik und Physik vol. 43 (1936) pp. 419-424.

2. K. Noshiro, Contributions to the theory of the singularities of analytic functions, Jap. J. Math. vol. 19 (1948) pp. 299-327.

3. G. P6lya and G. Szegö, Über den transfiniten Durchmesser (Kapazitätskonstante) von ebenen und räumlichen Punktmengen, J. Reine Angew. Math. vol. 165 (1931) pp. 4-49.

MASSAChUSETtS INSTITUTE OF TEChNOLOGY 